\title{
Tachyon Warm-Intermediate Inflationary Universe Model in High Dissipative Regime
}

\author{
M. R. Setare 1 \\ V. Kamali2 \\ Department of Science, Payame Noor University, Bijar, Iran
}

\begin{abstract}
We consider tachyonic warm-inflationary models in the context of intermediate inflation. We derive the characteristics of this model in slow-roll approximation and develop our model in two cases, 1- For a constant dissipative parameter $\Gamma$. 2- $\Gamma$ as a function of tachyon field $\phi$. We also describe scalar and tensor perturbations for this scenario. The parameters appearing in our model are constrained by recent observational data. We find that the level of non-Gaussianity for this model is comparable with non-tachyonic model.
\end{abstract}

\footnotetext{
${ }^{1}$ rezakord@ipm.ir

${ }^{2}$ vkamali1362@gmail.com
} 


\section{Introduction}

Big Bang model have many long-standing problems (horizon, flatness,...). These problems are solved in the framework of the inflationary universe models [1. Scalar field as a source of inflation provides the causal interpretation of the origin of the distribution of large scale structure and observed anisotropy of cosmological microwave background (CMB) [2]. In standard models for inflationary universe, the inflation period is divided into two regimes, slow-roll and reheating epochs. In slow-roll period kinetic energy remains small compared to the potential terms. In this period, all interactions between scalar fields (inflatons) and other fields are neglected and the universe inflates. Subsequently, in reheating period, the kinetic energy is comparable to the potential energy and inflaton starts an oscillation around the minimum of the potential losing their energy to other fields present in the theory. So, the reheating is the end period of inflation.

In warm inflationary models radiation production occurs during inflationary period and reheating is avoided [3]. Thermal fluctuations may be obtained during warm inflation. These fluctuations could play a dominant role to produce initial fluctuations which are necessary for Large-Scale Structure (LSS) formation. So the density fluctuation arises from thermal rather than quantum fluctuation [4]. Warm inflationary period ends when the universe stops inflating. After this period the universe enters in radiation phase smoothly [3. Finally, remaining inflatons or dominant radiation fields created the matter components of the universe.

Friedmann-Robertson-Walker (FRW) cosmological models in the context of string/M-theory have related to brane-antibrane configurations [5]. Tachyon fields associated with unstable D-branes may be responsible for inflation in early time [6]. If the tachyon field start to roll down the potential, then universe dominated by a new form of matter will smoothly evolve from inflationary universe to an era which is dominated by a non-relativistic fluid [7. So, we could explain the phase of acceleration expansion (inflation) in term of tachyon field.

In this work we would like to consider the tachyon warm inflationary universe in the particular scenario "intermediate inflation" which is denoted by scale factor $a(t)=a_{0} \exp \left(A t^{f}\right), 0<f<1[8]$. The expansion of this model is faster than power-law inflation $\left(a=t^{p} ; p>1\right)$, but slower than standard de sitter inflation $(a=\exp (H t))$. In non-tachyonic inflation, potential has the form $\phi^{-4 \frac{1-f}{f}}$, but in the warm-tachyon inflation we will receive to this form of potential $\phi^{-4 \frac{f-1}{2 f-1}}$. The Harrison-Zeldovich spectrum of density pertur- 
bation (i.e. $n_{s}=1[8]$ ) for these two cases are given by $f=\frac{2}{3}$. The study of intermediate inflationary model is motivated by string/M-theory [9]. In term of this theory, if the high order curvature corrections to Einstein-Hilbert action are proportional to the Gauss-Bonnet(GB) term, we obtain a free-ghost action. The GB term is the leading order of the $\alpha$ (inverse string tension) expansion to the low-energy string effective action [10]. This kind of theory is applied for study of initial singularity problem [11, black hole solutions [12] and the late time universe acceleration [13]. The coupling of GB with dynamical dilatonic scalar field in $4 D$ dark energy model leads to an intermediate form for scale factor, where $f=\frac{1}{2}, A=\frac{2}{8 \pi G n}$ with a constant parameter "n" 9]. So, intermediate inflation model may be derived from an effective theory at low dimensions of a fundamental string theory.

The tachyon inflation is a k-inflation model [14 for scalar field $\phi$ with a positive potential $V(\phi)$. Tachyon potentials have two special properties, firstly a maximum of these potential is obtained where $\phi \rightarrow 0$ and second property is the minimum of these potentials is obtained where $\phi \rightarrow \infty$. In our intermediate model we find an exact solution for tachyon field potential in slow-roll approximation which have the form $V(\phi) \sim \phi^{-\beta}$. This form of potential has the above properties of tachyon field potential. This special form of potential is everlasting, so we consider our model in the context of the warm inflation to bring an end for inflation period. We know that in the warm inflationary models, dissipative effect arises from a friction term. This effect could describe the mechanisms that scalar fields decay into a thermal bath via its interaction with other fields and finally warm inflation ends when the universe heats up to become radiation dominant. After inflation period the universe smoothly connected with the radiation Big Bang phase. Intermediate inflation in standard gravity with the presence of tachyon field has been considered in Ref.[15]. Also, the warm tachyon inflation in standard gravity has been studied in [16. As far as, we know, a model in which warm tachyon inflation in the context of intermediate inflation has not been yet considered.

The paper is organized as follows. In section II, we give a brief review about tachyon warm inflationary universe. In section III we consider high dissipative warm-intermediate inflationary phase in two cases 1- A constant dissipative parameter $\Gamma$. 2- dissipative parameter $\Gamma$ as a function of tachyon field $\phi$. In this section we also, investigate the cosmological perturbations. In section IV we consider the possibility of having non-gaussianity for warmtachyon model. Finally in section V, we close by concluding remarks. 


\section{Tachyon warm inflationary universe}

Tachyonic inflation model in a spatially flat Friedmann Robertson Walker (FRW) is described by an effective fluid which is recognized by energymomentum tensor $T_{\nu}^{\mu}=\operatorname{diag}\left(-\rho_{\phi}, p_{\phi}, p_{\phi}, p_{\phi}\right)$ [7], where energy density $\rho_{\phi}$, and pressure for the tachyon field are defined by

$$
\begin{aligned}
\rho_{\phi} & =\frac{V(\phi)}{\sqrt{1-\phi^{2}}} \\
p_{\phi} & =-V(\phi) \sqrt{1-\phi^{2}},
\end{aligned}
$$

respectively, where $\phi$ denotes the tachyon scalar field and $V(\phi)$ is the effective scalar potential associated with the tachyon field. Characteristics of any tachyon field potential are $\frac{d V}{d \phi}<0$ and $V(\phi \rightarrow 0) \rightarrow 0$ [17. Friedmann equation for spatially flat universe and conservation equation, in the warm tachyon inflationary scenario are given by [16]

$$
\begin{gathered}
H^{2}=\frac{8 \pi}{3 m_{p}^{2}}\left[\rho_{\phi}+\rho_{\gamma}\right], \\
\dot{\rho}_{\phi}+3 H\left(\rho_{\phi}+p_{\phi}\right)=-\Gamma \dot{\phi}^{2} \Rightarrow \frac{\ddot{\phi}}{1-\dot{\phi}^{2}}+3 H \dot{\phi}+\frac{V^{\prime}}{V}=-\frac{\Gamma}{V} \sqrt{1-\dot{\phi}^{2}} \dot{\phi},
\end{gathered}
$$

and

$$
\dot{\rho}_{\gamma}+4 H \rho_{\gamma}=\Gamma \dot{\phi}^{2}
$$

where $H=\frac{\dot{a}}{a}$ is the Hubble factor, $a$ is a scale factor, $m_{p}$ represents the Planck mass and $\rho_{\gamma}$ is the energy density of the radiation. Dissipative coefficient $\Gamma$ has the dimension $m_{p}^{5}$. In the above equations dots "." mean derivative with respect to time and prime "'" is derivative with respect to $\phi$. During the inflationary epoch the energy density (10) is the order of the potential $\rho_{\phi} \sim V$. Tachyon energy density $\rho_{\phi}$ in this era dominates over the energy density of radiation $\rho_{\phi}>\rho_{\gamma}$. In slow-roll regime $(\dot{\phi} \ll 1$ and $\ddot{\phi} \ll(3 H+\Gamma / V) \dot{\phi}[3])$ and when warm inflation radiation production is quasi-stable $\left(\dot{\rho}_{\gamma} \ll 4 H \rho_{\gamma}, \dot{\rho}_{\gamma} \ll \Gamma \dot{\phi}^{2}\right)$ the equations (2), (3) and (44) are reduced to

$$
H^{2}=\frac{8 \pi}{3 m_{p}^{2}} V
$$




$$
\begin{gathered}
3 H(1+r) \dot{\phi}=-\frac{V^{\prime}}{V}, \\
\rho_{\gamma}=\frac{\Gamma \dot{\phi}^{2}}{4 H},
\end{gathered}
$$

where $r=\frac{\Gamma}{3 H V}$. The main problem of inflation theory is how to attach the universe to the end of the inflation period. One of the solutions of this problem is the study of inflation in the context of warm inflation [18]. In this model radiation is produced during inflation period where its energy density is kept nearly constant. This is phenomenologically fulfilled by introducing the dissipation term, $\Gamma$, in the equation of motion as we have seen in Eq.(3). This term grants a continuous energy transfer from scalar field energy into a thermal bath. In this article we consider high dissipation regimen $(r \gg 1)$ where the dissipation coefficient $\Gamma$ is much greater than the $3 H \mathrm{~V}$. High dissipative and weak dissipative regime for non-tachyonic warm inflation have been considered in Ref.s [18] and [19] respectively. The main attention was given to the high dissipative regime. This regime is the more difficult of the two, and when high dissipative regime is understood, the weak dissipative regime could follow. Also, in Refs. [19] and [20], the study of warm inflation in the context of quantum field theory has been fulfilled in high dissipative regime. Dissipative parameter $\Gamma$ could be a constant or a positive function of $\phi$ by the second law of thermodynamics. In some works $\Gamma$ and potential of inflation have the same form [16], [21]. In Ref.[16], perturbation parameters for warm tachyon inflation have been obtained where $\Gamma=\Gamma_{0}=$ const and $\Gamma=\Gamma(\phi)=V(\phi)$. So, in this work we will study the intermediate tachyon warm inflation in high dissipative regime for these two cases.

From Eqs. (6) and (7) in high dissipation regimen we get

$$
\rho_{\gamma}=\frac{m_{p}^{2}}{32 \pi r}\left[\frac{V^{\prime}}{V}\right]^{2}
$$

We introduce the slow-roll parameter

$$
\epsilon=-\frac{\dot{H}}{H^{2}}=\frac{m_{p}^{2}}{16 \pi r}\left[\frac{V^{\prime}}{V}\right]^{2} \frac{1}{V} .
$$

Using Eqs. (8) and (9) in slow-roll regime $\left(\rho_{\phi} \sim V\right)$ we find a relation between the energy densities $\rho_{\phi}$ and $\rho_{\gamma}$ as

$$
\rho_{\gamma}=\frac{\epsilon}{2} \rho_{\phi}
$$


The second slow-roll parameter $\eta$ is given by

$$
\eta=-\frac{\ddot{H}}{H \dot{H}} \simeq \frac{m_{p}^{2}}{8 r V}\left[\frac{V^{\prime \prime}}{V}-\frac{1}{2}\left(\frac{V^{\prime}}{V}\right)^{2}\right] .
$$

The warm inflationary condition $\ddot{a}>0$ may be obtained by parameter $\epsilon$, satisfying the relation

$$
\epsilon<1 \text {. }
$$

From above equation and Eq.(10) the tachyon warm inflation epoch could take place when

$$
\rho_{\phi}>2 \rho_{\gamma} .
$$

Inflation period ends when $\epsilon \simeq 1$ which implies

$$
\rho_{\phi} \simeq 2 \rho_{\gamma} .
$$

The number of e-folds is given by

$$
N(\phi)=-\frac{8 \pi}{m_{p}^{2}} \int_{\phi_{1}}^{\phi_{2}} \frac{V^{2}}{V^{\prime}} r d \phi
$$

where $\phi_{1}$ and $\phi_{2}$ denote the begining and the end inflatons.

\section{Intermediate inflation}

In this section we consider high dissipative tachyon intermediate inflation. In intermediate inflation the scale factor follows the law

$$
a(t)=a_{0} \exp \left(A t^{f}\right) \quad 0<f<1,
$$

where $A$ is a positive constant with units $m_{p}^{f}$. We would like to consider our model in two important cases [16]. $1-\Gamma$ is a constant parameter. $2-\Gamma$ is a function of $\phi(\Gamma=f(\phi)=V(\phi))$

\section{$3.1 \Gamma=\Gamma_{0}=$ const}

Using Eqs. (5), (6) and (16) with $\Gamma=$ constant, we get tachyon scalar field $\phi$, and potential $V(\phi)$ as

$$
\phi=\sqrt{\frac{3 m_{p}^{2}(1-f)(f A)^{2}}{2 \pi \Gamma_{0}(2 f-1)^{2}}} t^{\frac{2 f-1}{2}},
$$


and

$$
V(\phi)=B_{1} \phi^{-\beta_{1}}
$$

where

$$
\beta_{1}=\frac{4-4 f}{2 f-1} \quad B_{1}=\frac{3 m_{p}^{2}(f A)^{2}}{8 \pi}\left(\frac{\Gamma_{0} 2 \pi(2 f-1)^{2}}{3 m_{p}^{2}(1-f)(f A)^{2}}\right)^{-\frac{\beta_{1}}{2}} .
$$

The Hubble parameter in term of $\phi$ becomes

$$
H(\phi)=\sqrt{\frac{8 \pi B_{1}}{3 m_{p}^{2}}} \phi^{-\frac{\beta}{2}}
$$

From equation (18), if $f>\frac{1}{2}, V(\phi)$ has the characteristic of tachyon field potential $\left(\frac{d V}{d \phi}<0\right.$ and $\left.V(\phi \rightarrow 0) \rightarrow 0\right)$, also these potentials do not have a minimum [22]. Slow-roll parameters $\epsilon$ and $\eta$ in term of tachyon field $\phi$ are obtained from Eqs. (9) and (11).

$$
\begin{gathered}
\epsilon=\sqrt{\frac{3 m_{p}^{2} B_{1}}{32 \pi \Gamma_{0}^{2}}} \beta_{1}^{2} \phi^{-\left(\frac{\beta}{2}+2\right)}, \\
\eta=\sqrt{\frac{3 m_{p}^{2} B_{1}}{32 \pi \Gamma_{0}^{2}}} \beta_{1}\left(\beta_{1}+2\right) \phi^{-\left(\frac{\beta}{2}+2\right)},
\end{gathered}
$$

respectively. From Eq.(15), the number of e-folds between two fields $\phi_{1}=$ $\phi\left(t_{1}\right)$ and $\phi_{2}=\phi\left(t_{2}\right)\left(\phi_{1}>\phi_{2}\right.$ if $\left.f>\frac{1}{2}\right)$ is given by

$N(\phi)=-\frac{8 \pi}{m_{p}^{2}} \int_{\phi_{1}}^{\phi_{2}} \frac{V^{2}}{V^{\prime}} \frac{\Gamma_{0}}{3 H V} d \phi=\frac{2 \Gamma_{0}}{\beta_{1}\left(\beta_{1}+4\right)} \sqrt{\frac{8 \pi}{3 m_{p}^{2} B_{1}}}\left(\phi_{2}^{\beta_{1} / 2+2}-\phi_{1}^{\beta_{1} / 2+2}\right)$

$\phi_{1}$ is obtained at the begining of inflation $(\epsilon=1), \phi_{1}^{-\left(\beta_{1} / 2+2\right)}=\sqrt{\frac{32 \pi \Gamma_{0}^{2}}{3 m_{p}^{2} B_{1}^{2}}} \frac{1}{\beta_{1}^{2}}$, so we can determine the value of $\phi_{2}$ in term of $N, A$ and $f$. At this stage we consider scalar and tensor perturbations for our model. For the tachyon field in warm inflationary universe (in slow-roll and high dissipative regime) the power spectrum of the curvature perturbation and amplitude of tensor perturbation (which would produce gravitational waves during inflation) are given by [16]

$$
P_{R} \simeq \frac{\sqrt{3}}{30 \pi^{2}} \exp (-2 \Im(\phi))\left[\left(\frac{1}{\epsilon}\right)^{3} \frac{9 m_{p}^{4}}{128 \pi^{2} r^{2} \sigma V}\right]^{\frac{1}{4}}
$$




$$
P_{T}=\frac{16 \pi}{m_{p}^{2}}\left(\frac{H}{2 \pi}\right)^{2} \operatorname{coth}\left[\frac{k}{2 T}\right] \simeq \frac{32 V}{3 m_{p}^{4}} \operatorname{coth}\left[\frac{k}{2 T}\right],
$$

respectively. Temperature $T$ in extra factor $\operatorname{coth}\left[\frac{k}{2 T}\right]$ denotes, the temperature of the thermal background of gravitational wave [23] and

$$
\Im(\phi)=-\int\left[\frac{1}{3 H r}\left(\frac{\Gamma}{V}\right)^{\prime}+\frac{9}{8} \frac{V^{\prime}}{V}\left[1-\frac{(\ln \Gamma)^{\prime}(\ln V)^{\prime}}{36 H^{2} r}\right]\right] d \phi .
$$

For $r \gg 1$ from Eqs.(24) and (25) tensor-scalar ration is obtained as

$$
\left.R\left(k_{0}\right) \approx \frac{240 \sqrt{3}}{25 m_{p}^{2}}\left[\frac{r^{\frac{1}{2}} \epsilon H^{3}}{T_{r}} \exp [2 \Im(\phi)] \operatorname{coth}\left(\frac{k}{2 T}\right)\right]\right|_{k=k_{0}} .
$$

$R$ is important parameter. We can use the seven-year Wilkinson Microwave Anisotropy Probe (WMAP7) observations to find an upper bound for $R$, from these results we have $P_{R} \simeq 2.28 \times 10^{-9}, R=0.21<0.36$ [2]. Spectral indices $n_{g}$ and $n_{s}$ were calculated in [16]

$$
\begin{aligned}
& n_{g}=-2 \epsilon \\
& n_{s}=1-\left[\frac{3}{2} \eta+\epsilon\left(\frac{2 V}{V^{\prime}}\left[2 \Im^{\prime}(\phi)-\frac{r^{\prime}}{4 r}\right]-\frac{5}{2}\right)\right] .
\end{aligned}
$$

In intermediate inflation, we obtained these parameters in term of tachyon field

$$
\begin{gathered}
n_{g}=-\sqrt{\frac{3 m_{p}^{2} \beta_{1}^{2}}{8 \pi \Gamma_{0}^{2}}} \beta_{1}^{2} \phi^{-\left(\beta_{1} / 2+2\right)} \\
n_{s}=1-\frac{3}{2} \eta-\frac{9}{4} \epsilon=1+\frac{3}{8}\left(\beta_{1}-4\right) \sqrt{\frac{\beta_{1}^{4} B_{1}}{\Gamma_{0}^{2} k}} \phi^{-\left(\beta_{1} / 2+2\right)} .
\end{gathered}
$$

Since $\frac{1}{2}<f<1$ we obviously see that the Harrison-Zeldovich spectrum (i.e, $n_{s}=1$ ) occurs for $\beta_{1}=4$ or equivalently $f=\frac{2}{3}$ which agrees with tachyon intermediate inflation [15] and non-tachyonic inflation model [8], 24]. $n_{s}>1$ is equivalent to $\beta_{1}>4$ and $n_{s}<1$ is equivalent to $\beta_{1}<4$. Runing in the spectral indices $\frac{d n_{s}}{d \ln k}$ is one of the important parameters which could be obtained by WMAP7 data [2].

$\alpha_{s}=\frac{d n_{s}}{d \ln k}=-\frac{d n_{s}}{d N}=-\frac{d \phi}{d N} \frac{d n_{s}}{d \phi}=-\frac{3 m_{p}^{2}\left(\beta^{2}-16\right)}{124 \pi} \frac{V^{\prime}}{V^{2} r} \sqrt{\frac{\beta_{1}^{4} B_{1}}{\Gamma_{0}^{2} k}} \phi^{-\left(\beta_{1} / 2+2\right)}$.

In terms of WMAP7 data, $\alpha_{s}$ is approximately -0.038 [2]. In the next subsection we will study the specific case in which the dissipative parameter is a function of $\phi$. 


\section{$3.2 \quad \Gamma=\Gamma(\phi)=V(\phi)$}

With $\Gamma=V(\phi)$ [16], and using the Eqs.(44) and (5), the slow-roll parameters become

$$
\begin{gathered}
\epsilon=\frac{\sqrt{3} m_{p} \beta_{2}^{2}}{2 \sqrt{8 \pi B_{2}}} \phi^{\beta_{2} / 2-2} \\
\eta=\frac{\sqrt{3} m_{p} \beta_{2}\left(\beta_{2}+2\right)}{2 \sqrt{8 \pi B_{2}}} \phi^{\beta_{2} / 2-2}
\end{gathered}
$$

where

$$
\beta_{2}=-4(f-1), \quad B_{2}=\frac{3 m_{p}^{2}(f A)^{2}}{8 \pi(2-2 f)^{4 f-4}}, \quad \phi=\sqrt{2(1-f) t} .
$$

The Hubble parameter and potential in this case are given by

$$
H(\phi)=\sqrt{8 \pi B_{2} / 3 m_{p}^{2}} \phi^{-\beta_{2} / 2}, \quad V(\phi)=B_{2} \phi^{-\beta_{2}},
$$

respectively. By using Eq.(15) the number of e-folds is obtained

$N(\phi)=\sqrt{8 \pi B_{2} / 3 m_{p}^{2}} \int_{\phi_{1}}^{\phi_{2}} \phi^{-\beta_{2} / 2+1} d \phi=\frac{2 \sqrt{8 \pi B_{2} / 3 m_{p}^{2}}}{\beta_{2}+4}\left(\phi_{2}^{-\beta_{2} / 2+2}-\phi_{1}^{-\beta_{2} / 2+2}\right)$.

$\phi_{1}$ is obtained at the begining of inflation $(\epsilon=1)$ and the value of $\phi_{2}$ could be determined in term of $N, A$ and $f$. Important parameters $R\left(k_{0}\right), n_{s}$ and $\alpha_{s}$ could be obtained in this case $(\Gamma=V)$ as

$$
\begin{gathered}
R\left(k_{0}\right)=\frac{48 \sqrt{3}}{5 m_{p}^{2}}\left[\left(\frac{16(8 \pi)^{2} \sigma^{2} \beta_{2}^{1} 2}{81\left(3 m_{p}^{2}\right)^{3} B_{2}^{8}}\right)\right] \phi^{\beta_{2}-\frac{7}{4}} \exp \left(\frac{\beta_{2}^{3}}{32 \sqrt{8 \pi B_{2} / 3 m p^{2}}\left(\beta_{2} / 2+2\right)} \phi^{-\beta_{2} / 2-2}\right)(35 \\
n_{s}=1-\frac{3}{2} \eta+\frac{3}{4} \epsilon(1+\epsilon)
\end{gathered}
$$

$\alpha_{s}=-\frac{d \phi}{d N} \frac{d n_{s}}{d \phi}=\frac{3 m_{p}^{2} \beta_{2}^{2}}{8 \pi B_{2}}\left(\frac{3 \sqrt{3} m_{p}}{8 \sqrt{2 \pi B_{2}}} \beta_{2}\left(\beta_{2}-4\right) \phi^{\frac{3}{2} \beta_{2}-5}-\left(\frac{3}{4} \beta_{2}+3\right)\left(\frac{\beta_{2}}{2}-2\right) \phi^{\beta_{2}-4}\right)$

respectively. These parameters may be constrained by WMAP7 data [2]. 


\section{Non-Gaussianity}

In this section we consider the possibility of having non-gaussianity for our model. So, we will obtain the bispectrum of the gravitational field fluctuations which are generated during warm-tachyon inflation period. The zero mode equation of motion of tachyon field $\phi$ have been obtain in Eq.(3). This

equation in slow-roll approximation and high-dissipative regime converts to the equation

$$
\frac{d \phi}{d t}=-\frac{1}{\Gamma} \frac{d V}{d \phi}
$$

In order to study the fluctuation of the thacyon field $\delta \phi(x, t)$, we need a local form of the above temporal or non-local equation [25]. We obtain this extended equation by imposing a near-thermal-equilibrium Markovian approximation. Therefore the fluctuation-dissipation theorem may be applied. From this the tachyon field is described by stochastic system which evolves according to a Langevin equation [26].

$$
\frac{d \phi}{d t}=\frac{1}{\Gamma u / V}\left[a^{-2} \nabla^{2} \phi-\frac{V^{\prime}}{V}+\frac{u \eta}{V}\right]
$$

where $\eta(x, t)$ is a stochastic source, and $u=\sqrt{1+\nabla_{\mu} \phi \nabla^{\mu} \phi}$. Using the fluctuation-dissipation theorem, the source term in momentum space has these properties

$$
\begin{aligned}
& <\eta>=0 \\
& <\eta(\mathbf{k}, t) \eta\left(\mathbf{k}^{\prime}, t^{\prime}\right)>=2 \Gamma T(2 \pi)^{3} \delta^{(3)}\left(\mathbf{k}-\mathbf{k}^{\prime}\right) \delta\left(t-t^{\prime}\right)
\end{aligned}
$$

Following the method which is used in Ref. [25], the full tachyon field is expressed as $\phi(x, t)=\phi_{0}(t)+\delta \phi(x, t)$, where $\phi_{0}$ is homogeneous background field and $\delta \phi \ll \phi_{0}$. In order to obtain the three-point correlation function of the density perturbation in Fourier space or the bispectrum, we consider the evolution equation up to second order fluctuations

$$
\delta \phi=\delta_{1} \phi+\delta_{2} \phi
$$

where $\delta_{1} \phi$ is in first order of $\delta \phi$ and $\delta_{2} \phi$ is in second order of $\delta \phi$. From Eq.(39), the evolution equations of Fourier modes of first and second order 
fluctuations are

$$
\begin{aligned}
\frac{d}{d t}\left(\delta_{1} \phi(\mathbf{k}, t)\right)= & \frac{1}{\Gamma}\left[-k^{2} V\left(\phi_{0}\right) \delta_{1} \phi(\mathbf{k}, t)-V^{\prime \prime} \delta_{1} \phi(\mathbf{k}, t)+\eta(\mathbf{k}, t)\right] \\
\frac{d}{d t}\left(\delta_{2} \phi(\mathbf{k}, t)\right)= & \frac{1}{\Gamma}\left[-k^{2} V\left(\phi_{0}\right) \delta_{2} \phi(\mathbf{k}, t)-V^{\prime \prime} \delta_{2} \phi(\mathbf{k}, t)\right. \\
& \left.-\left(\delta_{1} \phi(\mathbf{k}, t)\right)^{2}\left(V^{\prime} k^{2}+\frac{1}{2} V^{\prime \prime \prime}\right)\right]
\end{aligned}
$$

where these equations are obtained in a time period $t_{n}-t_{n-1}=\frac{1}{H}$ and we could obtain a complete solution over longer time by piecewise construction [25]. The solution of the above equations are

$$
\begin{aligned}
& \delta_{1} \phi(\mathbf{k}, t)=A\left(k, t-t_{n-1}\right) \int_{t_{n-1}}^{t} d t^{\prime} \frac{\eta\left(\mathbf{k}, t^{\prime}\right)}{\Gamma} A^{-1}\left(k, t^{\prime}-t_{n-1}\right) \\
& +A\left(k, t-t_{n-1}\right) \delta \phi_{1}\left(\mathbf{k} e^{-H\left(t_{n}-t_{n-1}\right)}, t_{n-1}\right)
\end{aligned}
$$

and

$$
\begin{aligned}
\delta \phi(\mathbf{k}, t)= & A\left(k, t-t_{n-1}\right) \int_{t_{n-1}}^{t} d t^{\prime} B\left(k, t^{\prime}\right) \\
& {\left[\int \frac{d p^{3}}{(2 \pi)^{3}} \delta_{1} \phi\left(\mathbf{p}, t^{\prime}\right) \delta_{1} \phi\left(\mathbf{k}-\mathbf{p}, t^{\prime}\right)\right] A^{-1}\left(k, t^{\prime}-t_{n-1}\right) } \\
& +A\left(k, t-t_{n-1}\right) \delta_{2} \phi\left(\mathbf{k} e^{-H\left(t_{n}-t_{n-1}\right)}, t_{n-1}\right)
\end{aligned}
$$

where

$$
\begin{gathered}
A(k, t)=\exp \left[-\int_{t_{0}}^{t}\left(\frac{k^{2} V\left(\phi_{0}\left(t^{\prime}\right)\right)}{\Gamma}+\frac{V^{\prime \prime}\left(\phi_{0}\left(t^{\prime}\right)\right)}{\Gamma}\right) d t^{\prime}\right] \\
B(k, t)=-\frac{2 V^{\prime}\left(\phi_{0}(t)\right) k^{2}+V^{\prime \prime \prime}\left(\phi_{0}(t)\right)}{\Gamma}
\end{gathered}
$$

The second term in solutions (43) and (44) are memory terms [27. The important concept of freeze-out is given by the relevance of these memory terms within the Hubble time. The freeze-out momentum $k_{F}$ is defined in momentum space as: When $k<k_{F}$ the memory term remainds during the Hubble time and for $k \geq k_{F}$ this term damps away. By definition $k_{F}$ have a condition

$$
\frac{V\left(\phi_{0}\right) k^{2}+V^{\prime \prime}\left(\phi_{0}\right)}{\Gamma H}>1
$$


In warm-inflation we have $V^{\prime \prime}\left(\phi_{0}\right)<\Gamma H$ and above condition may be simplified by this definition

$$
k_{F}=\sqrt{\frac{\Gamma H}{V\left(\phi_{0}\right)}}
$$

By using the solutions (43) and (44), the three-point function of the fluctuations at the large scale (when $t \simeq t_{60}$ or when the number of e-fold is equal to 60) will be computed before the end of inflation

$$
\begin{aligned}
& <\delta \phi\left(\mathbf{k}_{1}, t\right) \delta \phi\left(\mathbf{k}_{2}, t\right) \delta \phi\left(\mathbf{k}_{3}, t\right)>=A\left(k_{3}, t-t_{60}+1 / H\right) \\
& \int_{t_{60}-1 / H}^{t_{60}} A^{-1}\left(k_{3}, t^{\prime}-t_{60}+1 / H\right) B\left(k_{3}, t^{\prime}\right) \\
& {\left[\int \frac{d p^{3}}{(2 \pi)^{3}}<\delta_{1} \phi\left(\mathbf{k}_{1}, t\right) \delta_{1} \phi\left(\mathbf{p}, t^{\prime}\right)><\delta_{1} \phi\left(\mathbf{k}_{2}, t\right) \delta_{1} \phi\left(\mathbf{k}_{3}-\mathbf{p}, t^{\prime}\right)>\right]} \\
& \quad A\left(k_{3}, t-t_{60}+1 / H\right)<\delta_{1} \phi\left(\mathbf{k}_{1}, t_{60}\right) \delta_{1} \phi\left(\mathbf{k}_{2}, t_{60}\right) \delta_{2} \phi\left(\mathbf{k}_{3} e^{-1}, t_{60}-1 / H\right)> \\
& \quad+\left(\mathbf{k}_{1} \leftrightarrow \mathbf{k}_{3}\right)+\left(\mathbf{k}_{2} \leftrightarrow \mathbf{k}_{3}\right)
\end{aligned}
$$

In the freeze-out region $\left(k>k_{F}\right)$ and by using the approximations $A \simeq 1$ and $B(k, t) \approx B\left(k_{F}, t_{F}\right)$ the three-point function becomes

$$
\begin{aligned}
& <\delta \phi\left(\mathbf{k}_{1}, t\right) \delta \phi\left(\mathbf{k}_{2}, t\right) \delta \phi\left(\mathbf{k}_{3}, t\right)>\approx B\left(k_{F}, t_{F}\right) \Delta t_{F} \\
& {\left[\int \frac{d p^{3}}{(2 \pi)^{3}}<\delta_{1} \phi\left(\mathbf{k}_{1}, t\right) \delta_{1} \phi\left(\mathbf{p}, t^{\prime}\right)><\delta_{1} \phi\left(\mathbf{k}_{2}, t\right) \delta_{1} \phi\left(\mathbf{k}_{3}-\mathbf{p}, t^{\prime}\right)>\right.} \\
& \left.\quad+\left(\mathbf{k}_{1} \leftrightarrow \mathbf{k}_{3}\right)+\left(\mathbf{k}_{2} \leftrightarrow \mathbf{k}_{3}\right)\right] \quad \Delta t_{F}=t_{H}-t_{F} \approx \frac{1}{H} \ln \left(\frac{k_{F}}{H}\right)
\end{aligned}
$$

where $t_{F}$ is the time when the last three wavevectors thermalizes, and $t_{H}$ is the time at Hubble crossing of the smallest inflation perturbation modes. The bispectrum for slow-roll, single field is given by

$$
<\Phi\left(\mathbf{k}_{1} \mathbf{k}_{2} \mathbf{k}_{3}\right)>=2 f_{N L}(2 \pi)^{3} \delta^{3}\left(\mathbf{k}_{1}+\mathbf{k}_{2}+\mathbf{k}_{3}\right)\left[P_{\Phi}\left(\mathbf{k}_{1}\right) P_{\Phi}\left(\mathbf{k}_{2}\right)+\text { perms }\right]
$$

where the gravitational field $\Phi$ has the simple form

$$
\Phi(\mathbf{k})=-\frac{3}{5} \frac{H}{\dot{\phi}} \delta \phi(\mathbf{k})
$$

So, $f_{N L}$ for warm-tachyon inflation in high dissipative regime is

$$
f_{N L}=-\frac{5}{3}\left(\frac{\dot{\phi}}{H}\right)\left[\frac{1}{H} \ln \left(\frac{k_{F}}{H}\right)\left(\frac{V^{\prime \prime \prime}\left(\phi_{0}\left(t_{F}\right)\right)+2 k_{F}^{2} V^{\prime}\left(\phi_{0}\left(t_{F}\right)\right)}{\Gamma}\right)\right]
$$


In Ref. 25] the $f_{N L}$ parameter for non-tachyonic warm inflation is obtained as

$$
f_{N L}=-\frac{5}{3}\left(\frac{\dot{\phi}}{H}\right)\left[\frac{1}{H} \ln \left(\frac{k_{F}}{H}\right) \frac{V^{\prime \prime \prime}\left(\phi_{0}\left(t_{F}\right)\right)}{\Gamma}\right]
$$

For the same potential, we see the non-gaussianity in the curvature in warmtachyon inflation (52) is comparable to the non-tachyonic warm-inflation (53).

\section{Conclusion}

The study of inflationary epoch with intermediate scale factor leads to overlasting form of potential $\left(\phi^{-\beta}\right)$ which agrees with tachyon potential properties and the study of warm inflation model as a mechanism that gives an end for tachyon inflation are motivated us to consider the tachyon warmintermediate inflation model. So in this paper we have investigated the warm-tachyon-intermediate inflationary models. We have studied this scenario in two different cases of the dissipative coefficient $\Gamma$. Our model have been described for $\Gamma=\Gamma_{0}=$ const and for $\Gamma$ as a function of tachyon field $\phi$, i.e. $\Gamma=f(\phi)=V(\phi)$. For these cases we have found exact solutions of Friedmann for spatially flat universe containing tachyon scalar field $\phi(t)$. We also have obtained scalar potential and Hubble parameter as a function of tachyon field. In slow-roll approximation, explicit expressions for the tensor-scalar ratio $R$, scalar spectrum index $n_{s}$ and its running $\alpha_{s}$ have been obtained, we have constrained these parameters by WMAP7 observational data. In $\Gamma=\Gamma_{0}$ case, we introduced scalar field potential as $V(\phi) \propto \phi^{-4 \frac{f-1}{2 f-1}}, 0<f<1$, but in non-tachyonic inflation potential has the form $\phi^{-4 \frac{1-f}{f}}$. Tachyonic condition for potential $V(\phi)\left(\frac{d V}{d \phi}<0\right)$ constrained $f$ as $\frac{1}{2}<f<1$. In this case, it is possible in the slow-roll approximation to have the Harrison-Zeldovich spectrum of density perturbation (i.e. $n_{s}=1$ [8]), provided $f$ takes the value of $\frac{2}{3}$ which agrees with regular inflation model with a canonical scalar field characterized by a quasi-exponential expansion. We found The non-Gaussianity of warm tachyon model is comparable with non-tachyonic warm inflation.

\section{References}

[1] A. Guth, "The inflationary universe: A possible solution to the horizon and flatness problems," Phys. Rev. D 23, 347, (1981); A. Albrecht and 
P. J. Steinhardt, "Cosmology for grand unified theories with radiatively induced symmetry breaking," Phys. Rev. Lett. 48, 1220, (1982); A complete description of inflationary scenarios can be found in the book by A. Linde, "Particle physics and inflationary cosmology," (Gordon and Breach, New York, 1990).

[2] E. Komatsu et al., Seven-Year Wilkinson Microwave Anisotropy Probe (WMAP) Observations: Cosmological Interpretation, arXiv:1001.4538 [astro-ph.CO]; B. Gold et al., Seven-Year Wilkinson Microwave Anisotropy Probe (WMAP) Observations: Galactic Foreground Emission, arXiv:1001.4555 [astro-ph.GA]; D. Larson et al., Seven-Year Wilkinson Microwave Anisotropy Probe (WMAP) Observations: Power Spectra and WMAP-Derived Parameters, arXiv:1001.4635 [astroph.CO]; J. L. Weiland et al., Seven-Year Wilkinson Microwave Anisotropy Probe (WMAP) Observations: Planets and Celestial Calibration Sources, arXiv:1001.4731 [astro-ph.CO]; N. Jarosik et al., Seven-Year Wilkinson Microwave Anisotropy Probe (WMAP) Observations: Sky Maps, Systematic Errors, and Basic Results, arXiv:1001.4744 [astro-ph.CO]; C. L. Bennett et al., Seven-Year Wilkinson Microwave Anisotropy Probe (WMAP) Observations: Are There Cosmic Microwave Background Anomalies?, arXiv:1001.4758 [astroph.CO].

[3] A. Berera, "Warm inflation," Phys. Rev. Lett. 75, 3218, (1995); "Interpolating the stage of exponential expansion in the early universe: A possible alternative with no reheating," Phys. Rev. D 55, 3346, (1997).

[4] L. M. H. Hall, I. G. Moss and A. Berera, Phys.Rev.D 69, 083525 (2004); I.G. Moss, Phys.Lett.B 154, 120 (1985); A. Berera, Nucl.Phys B 585, 666 (2000).

[5] A. Sen, Rolling tachyon, JHEP 0204, 048, (2002).

[6] A. Sen, "Field theory of tachyon matter," Mod. Phys. Lett. A 17, 1797, (2002); M. Sami, P. Chingangbam and T. Qureshi, "Aspects of tachyonic inflation with exponential potential," Phys. Rev. D 66, 043530, (2002).

[7] G. W. Gibbons, "Cosmological evolution of the rolling tachyon," 2002 Phys. Lett. B 537, 1.

[8] J. D Barrow and A. R. Liddle, Phys. Rev. D 47, R5219 (1993); A. Vallinotto, E. J. Copeland, E. W. Kolb, A. R. Liddle and D. A. Steer, 
Phys. Rev. D 69, 103519 (2004); A. A. Starobinsky JETP Lett. 82, 169 (2005).

[9] A. K. Sanyal, Phys. Lett. B. 645, 1 (2007).

[10] T. Kolvisto and D. Mota, Phys. Lett. B 644104 (2007); Phys. Rev. D. 75023518 (2007).

[11] I. Antoniadis, J. Rizos and K. Tamvakis, Nucl. Phys. B 415497 (1994).

[12] S. Mignemi and N. R. Steward, Phys. Rev. D 475259 (1993).

[13] S. Nojiri, S. D. Odintsov and M. Sasaki, Phys. Rev. D 71123509 (2004); G. Gognola, E. Eizalde, S. Nojiri, S. D. Odintsov and E. Winstanley, Phys. Rev. D 73084007 (2006).

[14] C. Armendariz-Picon, T. Damour and V. Mukhanov, Phys. Lett. B. 458, 209 (1999).

[15] S. del Campo, R. Herrera, and A. Toloza, "Tachyon Field in Intermediate Inflation," Phys. Rev. D79, 083507, (2009).

[16] R. Herrera, S. del Campo, C. Campuzano, "Tachyon warm inflationary universe models," JCAP 0610, 009, (2006).

[17] A. Sen, JHEP 9808, 012 (1998).

[18] A. Berera and L.Z. Fang, Phys. Rev. Lett. 741912 (1995); H. P. de Oliveria and R. O. Ramos, Phys. Rev. D57 741 (1998); H. P. de Oliveira and S. E. Jors, Phys. Rev. D64 063513 (2001).

[19] A. Berera, M. Gleiser and R. O. Ramos, Phys. Rev. D58 123508 (1998); Phys. Rev. Lett. 83264 (1999).

[20] J. Yokoyama, A. Linde, Phys. Rev. D60, 083509 (1999); A. Berera and R. O. Ramos, Phys. Rev. D63 103509 (2001); A. Berera and T. W. Kephart, Phys.Lett. B456 (1999); Phys. Rev. Lett. 831084 (1999).

[21] M. A. Cid, S. del Campo and R. Herrera, "Warm inflation on the brane," JCAP 0710 (2007) 005 [arXiv:0710.3148 [astro-ph]].

[22] S. del Campo and R. Herrera, Phys. Rev. D 76, 103503 (2007).

[23] K. Bhattacharya, S. Mohanty and A. Nautiyal, "Enhanced polarization of CMB from thermal gravitational waves," Phys. Rev. Lett.97, 251301, (2006). 
[24] J. D. Barrow and A. R. Liddle, "Perturbation spectra from intermediate inflation," Phys. Rev. D 47, 5219 (1993) astro-ph/9303011].

[25] S. Gupta, A. Berera, A. F. Heavens and S. Matarrese, "Non-Gaussian signatures in the cosmic background radiation from warm inflation," Phys. Rev. D 66 (2002) 043510 astro-ph/0205152.

[26] E. Calzetta and B. L. Hu, "Nonequilibrium Quantum Fields: Closed Time Path Effective Action, Wigner Function and Boltzmann Equation," Phys. Rev. D 37, 2878 (1988).

[27] A. Berera, Nuclear Phys. B, 585, 666 (2000). 